\title{
Adecuación de las derivaciones desde Atención Primaria a un Servicio de Urología
}

\author{
Páez Borda A, Redondo González E, Ríos González E, Linares Quevedo A, Sáenz Medina J, \\ Castillón Vela I.
}

Servicio de Urología. Hospital de Fuenlabrada. Madrid.

Actas Urol Esp. 2007;31(10):1166-1171

\section{RESUMEN}

ADECUACIÓN DE LAS DERIVACIONES DESDE ATENCIÓN PRIMARIA A UN SERVICIO DE UROLOGÍA

Objetivo: Valorar indirectamente el grado de adhesión de los médicos de Atención Primaria (AP) a un protocolo de derivación consensuado con Atención Especializada (AE) sobre diez motivos de consulta urológica.

Material y métodos: Se analizaron todas las derivaciones al servicio de urología llevadas a cabo durante un período de 19 meses por 135 médicos adscritos a 10 centros de AP (CAP). El urólogo juzgó como "confome" o "no conforme al protocolo" la derivación. Se compararon los porcentajes de adecuación de las derivaciones desde cada CAP. Se exploraron las diferencias en la adecuación de las derivaciones procedentes de cada CAP en función del problema de salud. La relación entre el número de derivaciones y el número de canalizaciones adecuadas se exploró mediante un modelo de regresión lineal.

Resultados: El análisis tuvo lugar sobre 2841 derivaciones. El 57,2\% de esas derivaciones se ajustó al protocolo. No se detectaron diferencias significativas en los porcentajes de derivaciones 'conforme al protocolo' en relación a los CAP. Se detectaron diferencias significativas entre CAP en las derivaciones por cólico nefrítico e incontinencia urinaria. Se detectó una relación entre el número de pacientes remitidos y el número de remisiones "no conforme al protocolo" $\left(\mathrm{r}^{2}=0,86\right)$.

Conclusiones: La adecuación al protocolo fue escasa. Algunos problemas de salud deberían de ser revisados a nivel local; la mayoría de los motivos de derivación (de modo particular la microhematuria) deberían de revisarse en todos los CAP.

Palabras clave: Diagnóstico. Gestión clínica. Eficiencia Clínica.

\section{ABSTRACT}

ADEQUACY OF REFERRAL FROM PRIMARY CARE TO A DEPARTMENT OF UROLOGY

Objetive: To indirectly address the adequacy of referrals from general practitioners (GP) to specialized care taking into account a previously agreed protocol on ten urological topics.

Materials \& methods: The study analyzed all referrals to the Urology department originated in 10 primary care centres (135 GPs involved) throughout a 19-month period. Adequacy of 2841 referrals was checked. The urologist judged the referral as compliant (adequate) or not compliant (inadequate) with the terms of the protocol. Compliance per primary care centres was compared. Also referral adequacy corrected per centre and clinical topic was compared. The relationship between "absolute number of referrals" and "adequate referrals" was tested using a linear regression model.

Results: $57.2 \%$ of the referrals were inadequate. Overall, no significant differences were detected between primary care centres. Nevertheless significant differences between centres were evident in terms of referrals due to renal colic and female urinary incontinence. 70\% (94/135) of the GPs complied with the protocol in, at least, 50\% of the cases. A strong association between "absolute number of referrals" and "adequate referrals" was evident $\left(r^{2}=0.86\right)$.

Conclusions: Overall compliance with the protocol was modest. While no significant differences between centres were detected in terms of adequacy of referrals certain conditions have to be locally revisited; most of the topics (particularly microhematuria) have to be revisited in every center.

Keywords: Diagnostics. Clinical efficiency. Management. 
$\mathrm{L}$ a conexión entre las unidades de Atención Primaria (AP) y los servicios de Atención Especializada (AE) constituye una pieza fundamental en la organización de muchos sistemas de asistencia sanitaria. En el sistema sanitario español los médicos de AP (MAP) ejercen un importante papel de filtro en las derivaciones al nivel especializado (gatekeeping). Esa función no se da en otros sistemas de nuestro entorno socioeconómico.

La derivación de pacientes desde AP a los servicios de $\mathrm{AE}$ tiene importantes implicaciones para los pacientes y el sistema sanitario. Sin embargo, existe evidencia de que el proceso de derivación es subóptimo; se producen variaciones inexplicables en las tasas de derivación ${ }^{1}$ que sugieren que algunos pacientes se derivan de forma inadecuada, por lo que consumen recursos que podrían haberse utilizado para ofrecer otros servicios; además, algunos pacientes tratados de forma inapropiada en los ámbitos de AP podrían beneficiarse de la AE. También existe evidencia sobre lo inadecuado de los documentos de derivación asî como sobre la escasa comunicación en el momento de la derivación ${ }^{2}$.

A pesar de la creciente concienciación sobre los problemas asociados con las derivaciones, ha habido relativamente pocas investigaciones que evalúen las intervenciones para mejorar el mecanismo de derivación. Una revisión sistemática de los estudios relacionados con intervenciones profesionales para mejorar los mecanismos de derivación, estableció algunas conclusiones de tipo práctico $^{3}$; la formación continuada de los profesionales de AP junto con la creación de formularios de derivación estructurados ${ }^{4}$ y las sesiones conjuntas $^{5}$, fueron eficaces para mejorar la adecuación de las derivaciones. Sin embargo, el desarrollo y la difusión pasiva de directrices de consenso local (protocolización de los criterios de derivación) ${ }^{3,6}$ y la adopción de medidas de naturaleza financiera ${ }^{7}$ presentaron un escaso efecto.

Este estudio parte de la hipótesis de que la mera elaboración y difusión de un protocolo de derivación de pacientes no garantiza la adecuación de las derivaciones. El objetivo del presente análisis es valorar el grado de adhesión de los MAP a un protocolo de derivación consensuado entre AP y AE sobre diez motivos de consulta urológica de alta prevalencia ${ }^{8}$.

\section{MATERIAL Y METODOS}

Para este estudio se analizó la adecuación de 2841 derivaciones consecutivas (el 26,6\% del total de derivaciones al servicio de urología durante el período del estudio) a lo largo de un período de 19 meses (mayo 2005-noviembre 2006). El protocolo de derivación ${ }^{8}$ contempló los criterios para la derivación y las pruebas complementarias a incluir en el momento de la derivación de los pacientes afectados por once problemas de salud de alta prevalencia: cólico nefrítico, disfunción eréctil, dolor testicular, fimosis, incontinencia urinaria femenina, infertilidad, infección urinaria, masa escrotal, microhematuria, sintomas del tracto urinario inferior en el varón y planificación familiar.

En el momento de la visita el urólogo juzgó como "confome al protocolo" o "no conforme al protocolo" la derivación. Una vez liberalizadas las indicaciones para la vasectomía, no pareció oportuno el análisis de las derivaciones para planificación familiar.

Las derivaciones procedieron de 10 centros de AP. Ciento treinta y cinco (135) profesionales diferentes remitieron pacientes desde esos centros durante el período del estudio. Fue posible identificar el médico remitente en 2581 ocasiones (2581/2841, 90,8\%).

Asumiendo como posible causa para las diferencias en la adecuación de la derivación eventuales variaciones en el grado de difusión de los protocolos, se compararon los porcentajes de pacientes remitidos conforme al protocolo desde cada centro de AP (prueba de la chi cuadrado de Pearson). De modo similar, se exploraron las diferencias en la adecuación de las derivaciones procedentes de cada centro de AP en función del problema de salud (prueba de la chi cuadrado y test exacto de Fisher). Para posibilitar el análisis, los centros menos activos (centros 6 y 10) fueron excluidos de esta fase del estudio. De modo similar, y dado el escaso volumen de derivaciones como consecuencia de infertilidad, ese motivo de derivación fue también eliminado de esta fase del análisis. Dado el elevado número de profesionales implicados no fue posible un análisis matemático formal de las derivaciones en función del médico remitente. No obstante, se analizó gráficamente la adecuación. La relación entre el 
número absoluto de derivaciones $\mathrm{y}$ el número de canalizaciones conformes al protocolo se exploró mediante un modelo de regresión lineal.

Para todas las comparaciones se empleó un nivel de significación estadística del 95\%. El tratamiento de los datos se llevó a cabo con el programa estadístico SPSS versión 11.5.

\section{RESULTADOS}

La Tabla 1 recoge los motivos para la derivación y los porcentajes de derivaciones "conformes al protocolo" y "no conformes al protocolo". Globalmente, el $57,2 \%$ de las derivaciones se ajustó a lo protocolizado. No se detectaron diferencias significativas en los porcentajes de derivaciones"conformes al protocolo" $(\mathrm{chi}=0,17)$ en relación a los centros solicitantes (Tabla 2).

En relación a los problemas de salud analizados, se detectaron diferencias significativas $(\mathrm{chi}<0,05)$ entre los centros en lo referente a las derivaciones por cólico nefrítico e incontinencia urinaria femenina.

El 70\% (94/135) de los profesionales que derivaron pacientes al servicio de urología lo hicieron de modo adecuado al protocolo en al menos el $50 \%$ de las ocasiones.

Tabla 1. Total de derivaciones y porcentaje de derivaciones "conforme" y "no conforme" al protocolo consensuado.

\begin{tabular}{lccc}
\hline $\begin{array}{l}\text { Motivo derivación } \\
\text { (problema de salud) }\end{array}$ & \multicolumn{2}{c}{$\begin{array}{c}\text { Adecuación de } \\
\text { la derivación }\end{array}$} & Total \\
\cline { 2 - 3 } & Conforme & $\begin{array}{c}\text { No } \\
\text { conforme }\end{array}$ \\
\hline Cólico nefrítico & $185(67,3)$ & $90(32,7)$ & 275 \\
Disfunción eréctil & $144(50,7)$ & $140(49,3)$ & 284 \\
Dolor testicular & $199(64,4)$ & $110(35,6)$ & 309 \\
Fimosis & $358(79,6)$ & $92(20,4)$ & 450 \\
Incontinencia urinaria & $93(60,0)$ & $62(40,0)$ & 155 \\
femenina & $42(75,0)$ & $14(25,0)$ & 56 \\
Infertilidad & $161(62,2)$ & $98(37,8)$ & 259 \\
Infección urinaria & $89(65,4)$ & $47(34,6)$ & 136 \\
Masa escrotal & $74(35,7)$ & $133(64,3)$ & 207 \\
Microhematura & $279(39,3)$ & $431(60,7)$ & 710 \\
STUI & $\begin{array}{c}1624 \\
(57,2)\end{array}$ & $\begin{array}{c}1217 \\
(42,8)\end{array}$ & $\begin{array}{c}2841 \\
(100,0)\end{array}$ \\
Total & & & \\
\hline
\end{tabular}

Entre paréntesis, \%

STUI: síntomas del tracto urinario inferior en el varón.
Tabla 2. Total de derivaciones y porcentaje de derivaciones "conforme" y "no conforme" al protocolo consensuado en función del centro de procedencia de la derivación.

\begin{tabular}{lcccc}
\hline $\begin{array}{l}\text { Centro de } \\
\text { procedencia }\end{array}$ & \multicolumn{4}{c}{ Adecuación derivación } \\
\cline { 2 - 5 } & No Conforme & Conforme & Total & Valor p \\
\hline Centro 1 & $167(53)$ & $148(47)$ & 315 & \\
Centro 2 & $211(59,6)$ & $143(40,4)$ & 354 & \\
Centro 3 & $206(55,7)$ & $164(44,3)$ & 370 & \\
Centro 4 & $319(56,6)$ & $245(43,4)$ & 564 & \\
Centro 5 & $93(55,4)$ & $75(44,6)$ & 168 & \\
Centro 6 & $19(43,2)$ & $25(56,8)$ & 44 & 0,17 \\
Centro 7 & $158(55,6)$ & $126(44,4)$ & 284 & \\
Centro 8 & $285(59,3)$ & $196(40,7)$ & 481 & \\
Centro 9 & $143(63,8)$ & $81(36,2)$ & 224 & \\
Centro 10 & $23(62,2)$ & $14(37,8)$ & 37 & \\
Total & 1624 & 1217 & 2841 & $(100)$ \\
\hline
\end{tabular}

Entre paréntesis, \%

En lo referente a la posible relación entre el número de pacientes remitidos y la adecuación de la remisión, pareció existir una estrecha relación lineal entre el número de pacientes remitidos y el número de remisiones "no conformes al protocolo" $\left(r^{2}=0,86, p<0,001\right)$; en otras palabras, los médicos con mayor consumo de las prestaciones del servicio de urología no hicieron un uso más adecuado que los "pequeños consumidores". La Tabla 3 recoge aquellos problemas de salud en los que la adhesión al protocolo fue igual o inferior al $50 \%$.

\section{DISCUSION}

La conexión entre AP y AE constituye uno de los principales problemas organizativos de los sistemas sanitarios basados en la AP, como es el nacional. En otros sistemas sanitarios del entorno socioeconómico europeo (Francia, Bélgica, Alemania...) la función del MAP tiene otras características; entre otras diferencias, el MAP no ejerce de controlador del acceso a la AE. No obstante, la regulación de ese acceso constituye motivo de preocupación para cualquier organización sanitaria. Un reciente estudio sobre actuaciones para optimizar las derivaciones por patología ocular ${ }^{6}$ (for- 
Tabla 3. Problemas de salud en los que la adhesión al protocolo fue igual o inferior al 50\% (cuadros grises).

\begin{tabular}{|c|c|c|c|c|c|c|c|c|c|c|c|}
\hline & & \multicolumn{10}{|c|}{ Centros } \\
\hline & & 1 & 2 & 3 & 4 & $\mathbf{5}$ & 6 & 7 & 8 & 9 & 10 \\
\hline \multirow{10}{*}{ 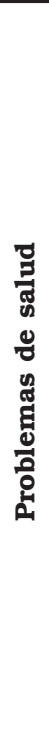 } & \begin{tabular}{|l} 
Cólico \\
nefrítico
\end{tabular} & & & & & & & & & & \\
\hline & $\begin{array}{l}\text { Disfunción } \\
\text { eréctil }\end{array}$ & & & & & & & & & & \\
\hline & $\begin{array}{l}\text { Dolor } \\
\text { testicular }\end{array}$ & & & & & & & & & & \\
\hline & Fimosis & & & & & & & & & & \\
\hline & $\begin{array}{l}\text { Incontinencia } \\
\text { urinaria } \\
\text { femenina }\end{array}$ & & & & & & & & & & \\
\hline & Infertilidad & & & & & & & & & & \\
\hline & $\begin{array}{l}\text { Infección } \\
\text { urinaria }\end{array}$ & & & & & & & & & & \\
\hline & $\begin{array}{l}\text { Masa } \\
\text { escrotal }\end{array}$ & & & & & & & & & & \\
\hline & Microhematuria & & & & & & & & & & \\
\hline & STUI & & & & & & & & & & \\
\hline
\end{tabular}

han detectado mejorias en la adecuación de las derivaciones. En un estudio aleatorizado sobre derivaciones por patología osteomuscular ${ }^{10}$ se observó una disminución significativa en las derivaciones a los servicios de cirugía ortopédica $(35,4 \%$ y $68,0 \%$, en los grupos a estudio y control, respectivamente, $p<0,001)$. En otro estudio se evaluaron los efectos de seminarios formativos dirigidos por especialistas, para divulgar entre médicos de AP directrices destinadas al tratamiento de la dispepsia ${ }^{6}$; sus autores plantearon la hipótesis de que la intervención aumentaría la adecuación de las derivaciones para exploraciones endoscópicas, aumentaría el rendimiento diagnóstico de las endoscopias, reduciría los gastos en antiácidos y aumentaría el número de solicitudes de pruebas

mación de los médicos de AP y generalización de los formularios específicos para la canalización) consiguió una reducción superior al 50\% en el número de derivaciones a servicios de oftalmología. Sin embargo, es muy difícil deducir qué aspecto de la intervención contribuyó en mayor medida al cambio en el número de derivaciones. En un estudio aleatorizado sobre adecuación de las derivaciones a $\mathrm{AE}$ motivadas por episodios de otitis media ${ }^{9}$, se evaluó el efecto de un vídeo divulgativo. En el grupo de médicos que tuvieron acceso al documento el porcentaje de derivaciones apropiadas aumentó después de la intervención. En una reciente experiencia sobre derivaciones a $\mathrm{AE}$ por enfermedades urológicas frecuentes (sintomas del tracto urinario inferior en varones, y hematuria microscópica $)^{4}$, se ofreció a los MAP participantes una reunión formativa, y se les envió por correo un paquete de directrices. La difusión entre los MAP de la existencia de una unidad de alta resolución mejoró el cumplimiento de las directrices de derivación de forma significativa.

Los estudios que han evaluado el efecto de las actividades formativas dirigidas por especialistas serológicas para Helicobacter pylori. Como consecuencia de esa intervención se produjeron tasas superiores de derivación para endoscopia, una mayor adecuación de las derivaciones, rendimientos diagnósticos ligeramente superiores y tasas más elevadas de pruebas serológicas. Sin embargo, no se produjo una reducción en la prescripción de antiácidos.

Otra línea de trabajo para mejorar la adecuación es el desarrollo de la "segunda opinión". Recientemente $^{11}$ se evaluó el efecto de una segunda opinión interna (dentro del ámbito de AP) antes de la derivación a las unidades de AE. Esa segunda opinión confirmó la necesidad de la derivación en el $70 \%$ de los casos, en tanto fue juzgada innecesaria en el 30\% de las ocasiones. Una alternativa a considerar es la teleconsulta ${ }^{13}$.

En nuestro medio, existen escasas experiencias de programas para la mejora de la adecuación de los protocolos de derivación de pacientes. En un análisis sobre las derivaciones desde las unidades de AP a un servicio de urgencias, la mitad de las canalizaciones $(49,4 \%)$ fueron juzgadas como inadecuadas ${ }^{12}$. De modo similar, un estudio reveló como innecesarias el $26 \%$ de las 
derivaciones a las especialidades médicas hospitalarias ${ }^{14}$. En la presente experiencia el $57,2 \%$ de las derivaciones fue considerado "conforme al protocolo". Uno de los problemas que se producen al juzgar las derivaciones es la definición de "adecuación". Existen iniciativas a este respecto ${ }^{15,16}$ pero siempre caben interpretaciones individuales de las directrices que convierten en estériles los intentos por protocolizar la derivación. En la presente experiencia los criterios para la derivación fueron consensuados entre AP y el Servicio de Urología con arreglo a la mejor evidencia clínica y atendiendo a las necesidades organizativas de ambos niveles, con lo que la probabilidad de derivaciones inadecuadas debería de resultar limitada. La modesta adhesión a los protocolos observada tampoco puede considerarse como producto de problemas en la accesibilidad al documento, dado que se encuentra disponible de modo universal y libre. Por ese motivo, resultan llamativas las diferencias observadas en la adecuación de las derivaciones como consecuencia de algunos problemas de salud (el cólico nefrítico y la incontinencia urinaria femenina, en concreto); probablemente se esté produciendo una diseminación insensible de las prácticas de unos profesionales a otros, que estaría sustituyendo a la consulta de los protocolos. Adicionalmente, la presión de los pacientes podría estar penalizando las decisiones de los MAP. Lo que resulta evidente es que la creación de documentos guía y su diseminación pasiva, no constituye un mecanismo fiable para la mejora de los mecanismos de derivación desde $\mathrm{AP}$ a $\mathrm{AE}$.

La principal limitación de este estudio se relaciona con el modo en que se decidió la adecuación de las derivaciones al protocolo; como ya se mencionó, esa decisión la tomó el urólogo que atendió al paciente derivado, valorando los términos de la derivación y las pruebas complementarias aportadas. Es posible que las calificaciones establecidas por los urólogos hubieran sido otras de haberlas llevado a cabo los MAP. Futuras evaluaciones del grado de adecuación deberán de incorporan la valoración de AP.

En la actualidad, los autores ultiman los detalles de un programa de divulgación urológica periódica entre los MAP, y desarrollan un sistema de información telefónica permanente pa- ra profesionales como medios para optimizar el funcionamiento armónico de los centros de AP y del Servicio de Urología.

\section{CONCLUSIONES}

- En definitiva, este estudio reveló una baja adhesión a los protocolos de trabajo consensuados entre $\mathrm{AP}$ y $\mathrm{AE}$ lo que confirma experiencias anteriores.

- Fue posible identificar problemas de salud que dieron lugar a diferencias inexplicables en la adecuación de las derivaciones (cólico nefrítico e incontinencia urinaria femenina), con independencia del centro origen de la derivación. Además, las derivaciones por microhematuria se produjeron de modo inadecuado en la mayor parte de las ocasiones. Probablemente los términos para algunas derivaciones deban de ser aclarados.

- Además, posibilitó la identificación de centros donde la adecuación de las derivaciones -con independencia de los problemas de salud- debería de mejorar globalmente.

Anexo: Trabajo parcialmente financiado con el Proyecto FIS P1070209.

\section{REFERENCIAS}

1. Wilkin D. Patterns of referral: explaining variation. Roland M, Coulter A, editor(s). Hospital Referrals. Oxford: Oxford University Press, 1992.

2. Roland MO, Coulter A. Hospital referrals. Oxford: Oxford University Press, 1992.

3. Grimshaw JM, Winkens RAG, Shirran L, Cunningham C, Mayhew A, Thomas R, Fraser C. Intervenciones para mejorar las derivaciones de pacientes ambulatorios desde la atención primaria a la atención secundaria (Revisión Cochrane traducida). En: La Biblioteca Cochrane Plus, número 4, 2006. Oxford, Update Software Ltd. Disponible en: http://www. update-software.com. (último acceso 27/11/06).

4. Thomas RE, Grimshaw JM, Mollison J, McClinton S, McIntosh E, Deans H, et al. Cluster Randomized Trial of a Guideline-Based Open Access Urological Investigation Service. Fam Pract. 2003;20(6):646-654.

5. Abu-Ramadan MA. Making better use of scarce resources: the Palestinian experience, 1995-1999. J Ambul Care Manage. 2002;25(3):63-69.

6. Jones RH, Lydeard S, Dunleavey J. Problems with implementing guidelines: a randomised controlled trial of consensus management of dyspepsia. Qual Health Care. 1993; 2(4):217-221.

7. Linnala A, Aromaa A, Mattila K. Specialists as consultants to GPs. Private sector services as an alternative way of organising consultant services in health care. Scand J Prim Health Care. 2001;19(2):90-94.

8. Protocolos de atención y derivación al paciente con enfermedad urológica. Atención Primaria Area 9. Hospital de Fuenlabrada. Disponible en http://10.35.122.139/intra-netA9/ index.asp (último acceso 27/11/06). 
9. Bennett K, Haggard M, Churchill R, Wood S. Improving referrals for glue ear from primary care: are multiple interventions better than one alone? J Health Serv Res Policy. 2001;6(3):139-44.

10. Vierhout WP, Knottnerus JA, van OOij A, Crebolder HF, Pop P, Wesselingh-Megens AM, et al. Effectiveness of joint consultation sessions of general practitioners and orthopaedic surgeons for locomotor system disorders. Lancet. 1995;346(8981):990-994.

11. Kinnersley P, Rapport FM, Owen P, Stott N. In-house referral: a primary care alternative to immediate secondary care referral?. Fam Pract. 1999;16(6):558-561.

12. E. Bouzas Senande, C. López Olmeda, E. Cerrada Cerrada, J. Olalla Linares, J. L. Menéndez. Adecuación de las derivaciones desde atención primaria al servicio de urgencias hospitalario en el Área 9 de Madrid. Emergencias 2005; 17 : 215-219.

13. Jaatinen PT, Aarnio P, Remes J, Hannukainen J, KöymäriSeilonen T. Teleconsultation as a replacement for referral to an outpatient clinic. J Telemed Telecare. 2002;8(2):102106.
14. M.J. García Pais MT, Rigueiro Veloso P, Rodríguez Ledo C, Rodríguez Fernández, A. Muriel, V. Abraira, B. Abarca, J. Conde, E. García Ferrero y E. Casariego Vales. Idoneidad de las derivaciones de Atención Primaria a especialidades médicas hospitalarias. SEMERGEN. 2006;32(8):376-381.

15. Knottnerus JA, Joosten J, Daams J. Comparing the quality of referrals of general practitioners with high and average referral rates: an independent panel review. Br J Gen Pract. 1990;40(334): 178-181.

16. Pringle M. Referral letters-ensuring quality. Practitioner. 1991;235(1503):507-510.

Correspondencia autor: Dr. A. Páez Borda Servicio de Urología. Hospital de Fuenlabrada Co del Molino, 2 - 28942 Fuenlabrada (Madrid) Tel.: 916006000

E-mail autor: apaez.hflr@salud.madrid.org Información artículo: Original - Gestión Trabajo recibido: enero 2007 Trabajo aceptado: julio 2007 